\title{
Parasitas da ordem Trypanorhyncha: sua importância na inspeção sanitária do pescado
}

\section{Parasites of the Trypanorhyncha order: importance in fished inspection}

\author{
Marcus de Freitas Ferreira, ${ }^{*}$ Sérgio Carmona de São Clemente, ${ }^{* *}$ Rogério Tortelly, ${ }^{* *}$ Francisco Carlos de Lima, ** \\ Elmiro Rosendo do Nascimento, ${ }^{* *}$ Geraldo Abreu de Oliveira, ${ }^{* *}$ Aline de Resende Lima****
}

\begin{abstract}
Resumo
Foram realizadas cento e cinqüenta necropsias em cinco espécies de peixes teleósteos de origem marinha comercializados no Mercado São Pedro, Município de Niterói, Rio de Janeiro. Utilizaram-se trinta exemplares para cada espécie de Pagrus pagrus, Pomatomus saltator, Priacanthus arenatus, Cynoscion sp. e Caranx latus, com o objetivo de verificar a prevalência e intensidade média de infecção de parasitas da ordem Trypanorhyncha. Com o auxílio de estereomicroscópio, os plerocercos foram liberados por meio de estiletes, colocados em placas de Petri com água e levados ao refrigerador por 24 horas. Após este tempo, foram processados segundo a técnica descrita por Amato et al. (1990) e identificados segundo Beveridge e Campbell (1994). As espécies Pagrus pagrus e Priacanthus arenatus estavam negativas para parasitas da ordem Trypanorhyncha. No gênero Cynoscion sp. foram observadas prevalência de $16,67 \%$ e média de intensidade de infecção de 0,17 parasitas por hospedeiro para Pterobotrium crassicolle. Em Caranx latus e Pomatomus saltator verificaram-se prevalência e intensidade média de infecção para Callitetrarhynchus gracilis de 33,33\% e 0,57 parasitas por hospedeiro e, de $36,67 \%$ e 0,60 parasitos por hospedeiro, respectivamente, sendo encontrado em $P$. saltator para $C$. speciosus prevalência de $3,33 \%$ e intensidade média de infecção de 0,03 parasitas por peixe. Os inspetores de pescado devem estar atentos à presença destes parasitas em peixes marinhos, pelo aspecto repugnante que transferem ao produto.
\end{abstract}

Palavras-chave:Trypanorhyncha, parasitas, peixes.

\begin{abstract}
A hundred and fifty necropsies have been done in five species in teleostean fishes in marine fishes commercialized in Niterói, Rio de Janeiro State. Were utilized thirty fishes for each species of Pagrus pagrus, Pomatomus saltator, Priacanthus arenatus, Cynoscion sp. and Caranx latus, with the objective of verifying the prevalence and the medium intensity infection of parasites from Trypanorhyncha order. The plerocercus were set free thru stiletto and set in Petri plate with water being kept under refrigeration per 24 hours. After this period, were processed following Amato's et al (1990) technics and identified following Beveridge and Campbell (1994). In Pagrus pagrus and Priacanthus arenatus were negatives for parasites from Trypanorhyncha order. In Cynoscion sp were found prevalence of $16,67 \%$ and medium infection intensity of 0,17 parasites per host for Pterobotrium crassicolle while in Caranx latus and Pomatomus saltator were found prevalence and medium infection intensity for Callitetrarhynchus gracilis of $33,33 \%$ and 0,57 parasites per host, as well, $36,67 \%$ and 0,60 parasites per host, respectively, being found in $P$. saltator for $C$. speciosus prevalence of $3,33 \%$ and medium infection intensity of 0,03 parasites per host. Fish sanitary inspectors must be aware of the presence of parasites from Trypanorhyncha order in marine fishes by the repugnant aspect that they transfer to the products.
\end{abstract}

Keywords: Trypanorhyncha, parasites, fishes.

\section{Introdução}

Os parasitos da ordem Trypanorhyncha podem ser considerados pouco estudados quando comparados com outros grupos de helmintos.
Os peixes parasitados por este grupo de cestóides são condenados pelo aspecto repugnante que apresentam. Segundo o Decreto № 30.691/52, em seu capítulo VII, seção I, que trata de pescados e derivados, no artigo 445, considerase impróprio para o consumo o pescado de aspecto

* Programa de Pós-Graduação em Medicina Veterinária (Doutorado). Higiene Veterinária e Processamento Tecnológico de Produtos de Origem Animal. Faculdade de Veterinária. Universidade Federal Fluminense - UFF.

** Departamento de Tecnologia dos Alimentos - Faculdade de Veterinária. Universidade Federal Fluminense - UFF - Rua Vital Brasil, 64, Santa Rosa, Niterói, RJ. CEP 24320-340.

*** Departamento de Saúde Coletiva Veterinária e Saúde Pública - Faculdade de Veterinária. Universidade Federal Fluminense, Niterói, RJ, Brasil.

**** Discente de Medicina Veterinária - UNIGRANRIO.

Autores para correspondência: Sérgio Carmona de São Clemente. E-mail: scsc@vm.uff.br e Marcus de Freitas Ferreira. E-mail: marcusff68@yahoo.com.br. 
repugnante, mutilado, traumatizado ou deformado, bem como que apresente infecção muscular maciça por parasitas, que possam prejudicar ou não a saúde do consumidor (Brasil, 1952).

Existem evidências que parasitas da ordem Trypanorhyncha ocasionam danos à saúde do consumidor. Rodero e Cuellar (1999) afirmam que estas larvas possuem componentes antigênicos capazes de provocar episódios anafiláticos, e que os produtos somáticos liberados pelas larvas de Gymnorhynchus gigas podem induzir o desenvolvimento de uma resposta imunitária humoral capaz de causar desordens alérgicas.

Resultados de Vázquez-López et al. (2000) demonstram que a ingestão de $G$. gigas pode produzir uma modulação da atividade colinérgica, originando importantes alterações da motilidade e do trânsito intestinal.

O objetivo do presente estudo foi verificar a prevalência e intensidade média de infecção dos parasitas da ordem Trypanorhyncha em cinco espécies de peixes teleósteos marinhos comercializados em Niterói, estado do Rio de Janeiro, e discutir a sua importância para a inspeção sanitária.

\section{Material e métodos}

Os peixes foram adquiridos no mercado São Pedro, no município de Niterói, estado do Rio de Janeiro, e conduzidos ao Laboratório de Inspeção e Tecnologia do Pescado da Faculdade de Veterinária da Universidade Federal Fluminense no período de setembro de 2005 a janeiro de 2006.

Realizaram-se 150 necropsias em cinco diferentes espécies, todas de origem marinha, sendo utilizados trinta exemplares para cada espécie. Foram estudados Pagrus pagrus, Pomatomus saltator, Priacanthus arenatus, Cynoscion sp. e Caranx latus.

Os dados relativos ao tamanho dos peixes e ao parasitismo foram registrados em fichas de necropsia individuais. As eviscerações foram realizadas a partir de um corte longitudinal ventral, da cloaca à região cefálica. Para a pesquisa do parasitismo na carne, os filés foram retirados e inspecionados com utilização da mesa de inspeção (Candling table).

Com o auxílio de estereomicroscópio, as larvas eram liberadas dos blastocistos por meio de estiletes e colocadas em placas de Petri com água e levadas ao refrigerador, onde permaneceram por 24 horas, para o relaxamento dos escólices e a extroversão dos tentáculos. Após serem retiradas do refrigerador, eram fixadas em A.F.A (Álcool 70\% formol - ácido acético) por, pelo menos, 24 horas, coradas pelo carmin de Langeron, diferenciadas pelo álcool clorídrico a $0,5 \%$, desidratadas em álcool, clarificadas pelo creosoto de Faia, e montadas entre lâmina e lamínula com bálsamo do Canadá, segundo a técnica descrita por Amato et al. (1990). Os parasitas foram identificados segundo Beveridge e Campbell (1994).

\section{Resultados e discussão}

Nas espécies Pagrus pagrus e Priacanthus arenatus não foram encontrados parasitas da ordem Trypanorhyncha. Em Cynoscion sp. foi registrada prevalência de 13,33\% e intensidade média de infecção de 0,17 parasitas por hospedeiro para Pterobotrium crassicolle. Em Caranx latus observaram-se prevalência e intensidade média de infecção de $33,33 \%$ e 0,57 para Callitetrarhynchus gracilis. Para Pomatomus saltator verificaram-se prevalência e intensidade média de infecção de $36,67 \%$ e 0,6 parasitas por hospedeiro para $C$. gracilis e de $3,33 \%$ e 0,03 parasitos por hospedeiro para Callitetrarhynchus speciosus, portanto, em relação a este peixe, a prevalência total para as espécies de Callitetrarhynchus foi de $40,00 \%$ e a média das intensidades de infecção foi de 0,63 parasitas por peixe.

Segundo dados de Palm (1997) para Pterobothrium kingstoni em Citharichthys spilopterus foi encontrada prevalência de $7,7 \%$, percentagem abaixo da encontrada no presente estudo para P. crassicolle. Lima (2004) encontrou para Pterobothrium crassicolle em Epinephelus guaza prevalência de 16,66\% e intensidade de infecção de uma larva por peixe, resultados superiores ao presente estudo com relação a prevalência. Já em Epinephelus niveatus, o referido autor encontrou prevalência de 3,33\% e intensidade de infecção de uma larva por peixe, índice de prevalência menor que o presente trabalho. Já Tavares (2006) obteve em Aspistor luniscutis prevalência de $11,6 \%$ e intensidade média de 1,1 parasitos por peixe, portanto, prevalência menor e intensidade média maior comparando com o presente estudo. São Clemente et al. (1997) encontraram em Pomatomus saltatrix prevalência de $7,5 \%$ e intensidade média de infecção de um plerocerco por peixe, portanto, prevalência menor e intensidade média acima do presente trabalho. Pereira Jr. et al.(2005) observaram em Pogonias cromis prevalência de $3,03 \%$, portanto abaixo do encontrado no presente estudo, e intensidade média de 1 parasita por peixe, acima da presente pesquisa e em Micropogonias furnieri prevalência de $66,67 \%$ e intensidade média de infecção de 5,15 parasitas por peixe, resultados muito acima comparando com o presente estudo. São Clemente (1986) pesquisando $M$. furnieri encontraram uma prevalência para Pterobotrium heteracanthum de $26 \%$ e intensidade média máxima de 8,2 parasitas/peixe, para Pterobotrium sp. obtiveram uma prevalência máxima de $23 \%$ e uma intensidade média máxima de 2,3 parasitos/peixe resultados muito acima dos encontrados para Pterobotrium crassicolle neste trabalho.

Lima (2004) relata o encontro de C. gracilis em Epinephelus. morio, com prevalência de $94 \%$, em Epinephelus guaza e Caranx crysos prevalência de $83,3 \%$ com intensidades médias de infecção de 59,2; 4,2 e 5 respectivamente, resultados acima dos encontrados para as espécies de peixes estudadas neste trabalho. Em Genypterus brasiliensis o mesmo autor encontrou prevalência de $75 \%$, em Epinephelus niveatus e Cynoscion acoupa 70\%, intensidades médias de infecção de 2,4; 54,7 e 5,6, respectivamente, resultados também acima dos aqui registrados. Em Epinephelus flavolimbatus e em Selene setapinnis as prevalências de 40 e $20 \%$ estão próximas de Caranx latus e Pomatomus saltator deste relato, enquanto as intensidades médias de 3,5 e 1 acima de todas as espécies por nós estudadas. Em Selene vômer a prevalência de $60 \%$ é acima da notada nesta pesquisa para Caranx latus com $33,33 \%$, bem como a intensidade média de infecção de 4,5. Em Priacanthus arenatus o autor registrou prevalência de $12,5 \%$ e intensidade média baixa de 1 parasita, enquanto que neste trabalho não foi encontrado parasita da ordem trypanorhyncha nesta espécie de peixe. São Clemente (1986), estudando Micropogonias furnieri, encontrou C. gracilis com 
prevalência máxima de $20 \%$ e uma intensidade média máxima de 2,3 parasitos/peixe, portanto, prevalência menor e intensidade média maior do que o presente trabalho. Alves e Luque (1999) observaram em Micropogonias furnieri prevalência de $1,3 \%$ e intensidade média de 1, portanto prevalência abaixo e intensidade média acima comparada ao presente estudo. Knoff et al. (2003) encontraram em Genypterus brasiliensis no Rio de Janeiro C. gracilis com prevalência de $18,2 \%$ e intensidade média de 3,3 parasitas por peixe, portanto, prevalência inferior e média de intensidade superior ao presente trabalho. Luque e Alves (2001) estudando Caranx hippos no litoral do estado do Rio de Janeiro encontraram prevalência de $1,6 \%$ e intensidade média de 1 parasita por peixe para $C$. gracilis, ou seja, prevalência também inferior e intensidade média pouco superior às encontradas no presente trabalho. Estes mesmos autores estudando o mesmo parasita em Caranx latus encontraram prevalência de $5,4 \%$ e intensidade média de 6,3 parasitas por peixe, ou seja, prevalência muito inferior e intensidade média muito superior à encontrada neste trabalho. Em estudo de Silva et al. (2000) foram observados para C. gracilis $14,5 \%$ de prevalência e intensidade média de infecção de 3,1 parasitas por peixe em Trichiurus lepturus do litoral do estado do Rio de Janeiro, índice de prevalência bem inferior e média de infecção um pouco superior ao presente estudo. Segundo dados de Palm (1997) pesquisando peixes do litoral brasileiro, foram encontrados $C$. gracilis em Caranx crysos com prevalência de $25 \%$, prevalência inferior às encontradas no presente estudo, porém segundo este autor o parasita não foi encontrado em Caranx latus, o que contradiz os achados deste trabalho. Ainda segundo Palm (1997), o mesmo parasita foi encontrado nas seguintes espécies de peixe com respectivas prevalências: Chloroscombrus chrysurus (23,5\%), inferior ao presente trabalho; Oligoplites palometa $(11,1 \%)$, idem análise anterior; Selene vomer $(50 \%)$, superior ao presente estudo; Harengula clupeola $(21,8 \%)$, inferior ao presente trabalho; Opisthonema oglinum $(34,8 \%)$, semelhante às encontradas no presente estudo; Haemulon aurolineatum (72\%), superior ao presente trabalho; Larimus breviceps $(7,7 \%)$, muito inferior ao presente estudo; Scomberomorus maculatus (12,5\%), inferior à encontrada no presente trabalho e Sphyraena guachancho (25\%), um pouco inferior à encontrada no presente estudo. Já Silva e São Clemente (2001) encontraram C. gracilis em Lutjanus synagris com prevalência de $0,71 \%$ e intensidade de infecção igual a 1 , ou seja, prevalência baixa em relação à encontrada em Pomatomus saltator neste trabalho. Timi et al. (2005) encontraram prevalência de $18,2 \%$ e intensidade média de infecção de 1,2 parasitos em Cynoscion guatucupa no Uruguai e na Argentina pesquisando a mesma espécie de peixe encontraram $21,3 \%$ de prevalência e 1,6 de intensidade média de infecção. Os dados de prevalência no Uruguai e na

\section{Referências}

ALVES, D.R.; LUQUE, J.L. Aspectos quantitativos das infrapopulações de metazoários parasitos de indivíduos jovens da corvina, Micropogonias furnieri (Osteichthyes: Sciaenidae) do litoral do estado do Rio de Janeiro, Brasil. Contribuições Avulsas sobre História Natural Brasileira, Ser. Zool., Seropédica: Universidade Federal Rural do Rio de Janeiro, v. 10, p. 1-4, dez. 1999.
Argentina estão abaixo dos encontrados no presente estudo, entretanto, os de intensidade média de infecção estão maiores. Alves (2001) no Brasil encontrou prevalência de 4\% e intensidade média de infecção de 1,2 parasitas por peixe em Micropogonias furnieri estando, portanto, os dados de prevalência menores e de intensidade média de infecção acima do presente estudo. Sabas e Luque (2003) encontraram em Macrodon ancylodon prevalência de $3 \%$ e intensidade média de 1 parasita por hospedeiro, análise pois semelhante à anterior. Já em Cynoscion guatucupa estes autores encontraram prevalência e intensidade média de infecção igual a zero, o que confirma os achados deste trabalho para Pagrus pagrus, Priacanthus arenatus e Cynoscion sp.

Para Callitetrarhynchus speciosus Lima (2004) encontrou em Epinephelus. morio prevalência de 5,8\% e intensidade média de infecção de duas larvas por peixe, em Epinephelus guaza, prevalência de $16,6 \%$ e intensidade média de infecção de uma larva por peixe, em Epinephelus flavolimbatus prevalência de $40 \%$ e intensidade média de infecção de 6 , em Epinephelus niveatus prevalência de $56,6 \%$ e intensidade média de infecção de 3,8 e em Priacanthus arenatus prevalência de $12,5 \%$ e intensidade média de infecção de uma larva por peixe. São considerados elevados tais percentuais, já que nesta pesquisa foi encontrado um $C$. speciosus em Pomatomus saltator, com prevalência de 3,33\% e intensidade média de infecção de 0,03 larvas por peixe. São Clemente et al. (1997) encontraram prevalência de $40 \%$ e intensidade média de 6,75 larvas por peixe em Pomatomus saltatrix, dados muito mais elevados do que os encontrados neste trabalho. Pereira Jr. et al. (2005) obtiveram em Cynoscion guatucupa prevalência de $11,43 \%$ e intensidade média de infecção de 2,17 parasitas por hospedeiro confirmando a comparação anterior. Knoff et al. (2003) encontraram em Genypterus brasiliensis prevalência de 1,8\% e intensidade de infecção de 1 parasita por hospedeiro para $C$. speciosus, ou seja, prevalência inferior e média de intensidade superior ao presente estudo.

As variações da prevalência e da intensidade média de parasitos da ordem Trypanorhyncha são dependentes possivelmente da idade e dos hábitos alimentares dos peixes, bem como da resposta imune do hospedeiro.

\section{Conclusão}

Apesar da alta prevalência dos parasitos da ordem Trypanorhyncha, a intensidade média por exemplar foi baixa. Entretanto, os inspetores sanitários devem estar alertas quanto à presença destes parasitas devido ao aspecto repugnante que transferem ao peixe, levando-o a sua rejeição pelo consumidor ou a condenação dos filés nas indústrias de pescado.

ALVES, D.R.; LUQUE, J.L. Community Ecology of the Metazoan Parasites of White Croaker, Micropogonias furnieri (Osteichthyes: Sciaenidae), from the Coastal Zone of the State of Rio de Janeiro, Brazil. Memórias do Instituto Oswaldo Cruz, Rio de Janeiro: Instituto Oswaldo Cruz, v. 96, n. 2, p. 145-153, fev. 2001.

AMATO, J.F.R.; SÃO CLEMENTE, S.C.; OLIVEIRA, GA. Tentacularia coryphaenae, Bosc, 1801 (Eucestoda: Trypanorhyncha) in the inspection and technology of the skipjack tuna, Katsuwonus pelamis (L.) (Pisces: Scombridae). Atlântica, v. 12, p. 73-77, 1990. 
BRASIL. Ministério da Agricultura. Secretaria Nacional de Defesa Agropecuária. Regulamento da Inspeção Industrial e Sanitária de Produtos de Origem Animal. Aprovado pelo Decreto ํㅜ 30.691, de 29/ 03/52. Brasília, DF, 1952.

BEVERIDGE, I.; CAMPBELL, R.A. Redescription of Diesingium lomentaceum (Diesing, 1850) (Cestoda: Trypanorhyncha). Systematic Parasitology, v. 27, p. 149-157, 1994.

KNOFF, M. et al. Helmintos de importância sanitária presentes no congro-rosa, Genypterus brasiliensis Regan, 1903, comercializados no estado do Rio de Janeiro. Higiene Alimentar, São Paulo: L. F. G. S. Higiene Alimentar Publicações e Serviços LTDA, v. 17, n. 104/105, p. 89-90, jan./fev. 2003.

LIMA, F.C. Cestóides da ordem trypanorhyncha em peixes teleósteos comercializados no estado do Rio de Janeiro. Niterói, 2004. Tese (Doutorado em Medicina Veterinária) - Faculdade de Veterinária, Universidade Federal Fluminense, Niterói. 2004.

LUQUE, J.L.; ALVES, D.R. Ecologia das comunidades de metazoários parasitos do xaréu, Caranx hippos (Linnaeus) e do xerelete, Caranx latus Agassiz (Osteichthyes, Carangidae) do litoral do estado do Rio de Janeiro, Brasil. Revista Brasileira de Zoologia, Curitiba: Sociedade Brasileira de Zoologia, v. 18, n. 2, p. 399-410, 2001.

PALM, H.W. Trypanorhynch cestodes of commercial fishes from northeast brazilian coastal waters. Memórias do Instituto Oswaldo Cruz, Rio de Janeiro: Instituto Oswaldo Cruz, v. 92, n. 1, p. 69-79, jan./ fev. 1997.

PEREIRA Jr., J.; BOEGER, W.A. Larval tapeworms (Platyhelminthes, Cestoda) from sciaenid fishes of the southern coast of Brazil. Zoosystema, Paris: Muséum National d'Histoire Naturelle, v. 27, n. 1 , 2005.

RODERO, M.; CUÉLLAR, C. Humoral responses induced by Gymnornhynchus gigas extracts in BALB/ c mice. Journal of Helminthology, v. 73, p. 239-273, 1999.

SABAS, C.S.S.; LUQUE, J.L. Metazoan parasites of weakfish, Cynoscion guatucupa and Macrodon ancylodon (Osteichthyes: Sciaenidae), from the coastal zone of the state of Rio de Janeiro,
Brazil. Revista Brasileira de Parasitologia Veterinária, v. 12, n. 4, p. 171-178, 2003.

SÃO CLEMENTE, S.C. Prevalência e intensidade média de infecção de plerocercos de Trypanorhyncha, parasitando corvina Micropogonias furnieri (Desmarest) no litoral do Rio de Janeiro. Atas Soc. Biol. RJ, Rio de Janeiro: Sociedade de Biologia do Rio de Janeiro, v. 26, p. 3744, 1986.

SÃO CLEMENTE, S.C.; SILVA, C.M.; GOTTSCHALK, S. Prevalencia e intensidade de infecção de cestóides trypanorhyncha em anchovas, pomatomus saltatrix (L.), do litoral do Rio de Janeiro, Brasil / Prevalence and intensity of the infection of trypanorhynch cestodes in bluefish, pomatomus saltatrix (L.), in the Rio de Janeiro coastline, Brazil. Parasitología al día, Santiago, v. 21, n. 1/2, p. 54-57, jun. 1997.

SILVA, C.M.; SÃO CLEMENTE, S.C. Nematóides da família anisakidae e cestóides da ordem trypanorhyncha em filés de dourado (Coryphaena hippurus) e ariocó (Lutjanus synagris) e sua importância na inspeção de pescado. Higiene Alimentar, v. 15, n. 80/81, p. 75-79, jan./fev. 2001.

SILVA, L.O. et al. Ecologia da comunidade de metazoários parasitos do peixe-espada Trichiurus lepturus Linnaeus (Osteichthyes, Trichiuridae) do litoral do estado do Rio de Janeiro, Brasil. Revista Brasileira de Zoociências, v. 2, n. 2, p. 115-133, dez. 2000.

TAVARES, L.E.R. Composição e estrutura das comunidades de metazoários parasitos de Aspistor luniscutis e Genidens barbus (Osteichthyes: Ariidae) e Anchoa marinii e A. tricolor (Osteichthyes: Engraulididae) do litoral do estado do Rio de Janeiro, Brasil. Seropédica, 2006. 133 p. Tese (Doutorado em Ciências Veterinárias) Instituto de Veterinária, Universidade Federal Rural do Rio de Janeiro, Seropédica. 2006.

TIMI, J.T.; LUQUE, J.L.; SARDELLA, N.H. Parasites of Cynoscion guatucupa along South American Atlantic coasts: evidence for stock discrimination. Journal of Fish Biology, v. 67, p. 1603-1618, 2005.

VÁZQUEZ-LÓPEZ, C. et al. Efffect of Gymnorhynchus gigas plerocercoid crude extract homogenate on the rat ileum motility in vitro. International Journal of Food Microbiology, 2000. 